\title{
Brain, emotion and decision making: the paradigmatic example of regret
}

\author{
Giorgio Coricelli ${ }^{1}$, Raymond J. Dolan ${ }^{2}$ and Angela Sirigu ${ }^{1}$ \\ ${ }^{1}$ Neuropsychology Group, Institut des Sciences Cognitives, CNRS, 67 Boulevard Pinel, 69675 Bron, France \\ ${ }^{2}$ Wellcome Department of Imaging Neuroscience, 12 Queen Square, London, WC1N 3BG, UK
}

\begin{abstract}
Human decisions cannot be explained solely by rational imperatives but are strongly influenced by emotion. Theoretical and behavioral studies provide a sound empirical basis to the impact of the emotion of regret in guiding choice behavior. Recent neuropsychological and neuroimaging data have stressed the fundamental role of the orbitofrontal cortex in mediating the experience of regret. Functional magnetic resonance imaging data indicate that reactivation of activity within the orbitofrontal cortex and amygdala occurring during the phase of choice, when the brain is anticipating possible future consequences of decisions, characterizes the anticipation of regret. In turn, these patterns reflect learning based on cumulative emotional experience. Moreover, affective consequences can induce specific mechanisms of cognitive control of the choice processes, involving reinforcement or avoidance of the experienced behavior.
\end{abstract}

\section{Introduction}

I should have computed the historical covariance of the asset classes and drawn an efficient frontier. Instead I visualized my grief if the stock market went way up and I wasn't in it - or if it went way down and I was completely in it. My intention was to minimize my future regret, so I split my [pension scheme] contributions $50 / 50$ between bonds [risk-free assets] and equities [risky assets].

This quotation describes a common pattern of vacillation in behavior, which is all the more striking considering it was formulated by Harry Markowitz (as reported in Money magazine in January 1998), the 1990 Nobel Prize winner for pioneering work in the theory of financial economics. Markowitz is explicit in his admission of making less-thanoptimal choices in relation to his pension investment ('hedge away from the extremes'), to avoid the prospect of future regret.

Regret is an emotion associated with a decision that turns out badly. It is classically elicited by a comparison (counterfactual [1-3]) between the outcome of a choice (reality) and the better outcome of foregone rejected alternatives (what might have been). Regret embodies the painful lesson that things would have been better under a different choice, thus inducing a disposition to behavioral

Corresponding authors: Coricelli, G. (coricelli@isc.cnrs.fr); Dolan, R.J (rdolan@fil.ion.ucl.ac.uk); Sirigu, A. (sirigu@isc.cnrs.fr).

Available online 1 May 2007. change. People, including those with a deep knowledge of optimal strategies, such as Markowitz, often try to avoid the likelihood of future regret, even when this conflicts with the prescription of decisions based on rational choice; according to the latter, individuals faced with a decision between multiple alternatives under uncertainty will opt for the course of action with maximum expected utility, a function of both the probability and the magnitude of the expected payoff [4].

Here, we outline, for the first time, the neural basis of the emotion of regret, and its fundamental role in adaptive behavior. The following questions will be addressed: what are the neural underpinnings of 'powerful' cognitively generated emotions such as regret? What are the theoretical implications of incorporating regret into the process of choice, and into adaptive models of decision making? In line with recent work on emotion-based decision making [5,6], we attempt to characterize the brain areas underlying decision processes under risk and, more specifically, specify the functional relationship between 'rational' decision making and emotional influences that affect these decisional processes. Our focus, by way of illustration, is on the contribution of the orbitofrontal cortex (OFC) to both the

\section{Glossary}

Belief learning (Bayesian learning in games): concept used in game theory to indicate learning through updating of existing beliefs about other players' strategies in a repeated game. At any stage of the game, a player's prior beliefs are updated in light of new evidence using Bayes' rule. The rule follows the mathematical law of conditional probabilities - that is, probabilities conditional on observing an event.

Correlated equilibrium: a game-theoretical solution concept (a refinement of Nash equilibrium) introduced by Aumann [64]. A correlated equilibrium arises when players choose their actions according to a set of received signals or instructions (which do not affect the payoffs of the game). The context of the correlated equilibrium is one in which there is a 'device' (or referee) that assigns (privately) play instructions to the players of a game. The equilibrium is a probability distribution of those instructions providing the best response (rational) to the other players' instruction-based actions. Nash equilibrium: the most important solution concept in game theory [65]. It implies that each player's strategy is a best response (payoff maximizing) to the strategies that he predicts the other players will use. Such prediction requires that rationality is mutually known, 'I know that you know that I am rational, and so on'. Utility function: a mathematical representation of an individual's preferences over alternative outcomes. With a utility function, we can turn a choice problem into a numerical maximization problem [66]. 
Box 1. The Allais [51] paradox ('common consequence effect')

Consider the choices (first choice: A versus $B$; and second choice: $C$ versus $D$ ) between lotteries in Table I. For instance, if you choose lottery $A$ you can win $\$ 2500$ if a number from 1 to 33 is drown from a deck of numbered cards from 1 to 100 (i.e. with $33 \%$ probability), $\$ 0$ if number 34 is drawn (i.e. $1 \%$ probability) and $\$ 2400$ if a number from 35 to 100 is drawn (i.e. 66\% probability), and so on. 'Preferences' indicate the percentage of observed choices [52].

The choice pattern (actual preferences) $\mathrm{B}>\mathrm{A}$ and $\mathrm{C}>\mathrm{D}$ violates expected utility theory (EUT) [4]. EUT predicts that you should choose the lottery with the maximum expected utility, calculated as the sum of the utilities of each possible outcome, weighted by their probabilities. In our example, the first choice, B $>A$, implies, $\mathrm{u}(2400)>0.33 \mathrm{u}(2500)+0.1 \mathrm{u}(0)+0.66 \mathrm{u}(2400)$ (where ' $\mathrm{u}$ ' is utility) or, equivalently, $0.34 u(2400)>0.33 u(2500)$, whereas the second choice, C $>$ D, implies $0.33 \mathrm{u}(2500)>0.34 \mathrm{u}(2400)$. We assumed $\mathrm{u}(0)=0$, for simplicity. An Allais paradox occurs when the addition of an independent event influences choice behavior. In the case Table l, we add $\$ 2400$ to each option of the first choice and $\$ 0$ for the second choice (grey column). Without the grey column (common consequence), the choice between $A$ and $B$ is identical to the choice between $C$ and $D$. For the expected utility ('independence axiom'), adding the grey column should not have affected behavior. This paradoxical behavior can be explained by anticipated regret $[10,11]$, by which the choice of the option $A$ is avoided owing to the expected emotional reaction (regret) that one would experience if the outcome $\$ 0$ results.

Table I. The poor man's version of the Allais paradox ${ }^{a}$

\begin{tabular}{|lllll|}
\hline Lottery & $\mathbf{1 - 3 3}$ & $\mathbf{3 4}$ & $\mathbf{3 5 - 1 0 0}$ & Preferences \\
\hline A & 2500 & 0 & 2400 & $18 \%$ \\
B & 2400 & 2400 & 2400 & $82 \%$ \\
C & 2500 & 0 & 0 & $83 \%$ \\
D & 2400 & 2400 & 0 & $17 \%$ \\
\hline
\end{tabular}

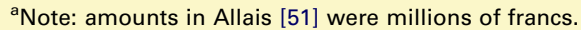

experience and anticipation of regret. We present a new hypothesis, based on regret theory, that we suggest can explain decision-making dysfunctions observed in OFClesioned patients, and account for the relationship between cognitive and emotional influences during decision making.

\section{The psychology of regret: top-down relationship between cognition and emotion}

Regret results when the outcome of a decision is compared with a better outcome within rejected alternatives [7]. This type of feedback information is crucial to determine the nature of the emotional response [8]. Norm theory, as articulated by Kahneman and Miller [9], suggests that the norms used in outcome evaluation are assessed after the outcome actually occurs. The norm acts as an appropriate context - a point of reference-used in the evaluation processes. This psychological perspective postulates that an outcome automatically evokes alternatives for comparison, in terms of what could or might have been. The norms of what might have happened if a person had chosen differently evoke a strong affective reaction. Thus, regret is quintessentially a cognitive-based emotion characterized by a feeling of responsibility for the negative outcome of a choice [10-12].

\section{Regret theory}

There are several economic models of regret. Classical early examples are the models of Loomes and Sugden [11], and Bell [10], who suggested that incorporating regret into the utility function (see Glossary) might reconcile utility theory with observed behavior (Allais paradox types of behavior; Box 1) during decision making under uncertainty. Therefore, many violations of the axioms of von Neumann and Morgenstern [4] expected utility theory might, in principle, be explained by the influence of anticipated regret. A decision maker under such influences might incur a suboptimal choice to avoid future regrettable situations. There are two key points within regret theory: first, the fact that regret is commonly experienced and, second, people try to anticipate and avoid the experience of future regret [11]. Anticipated regret is based on considering choosing an alternative and simultaneously rejecting other alternatives. In this theoretical setting, regret is not fully rational. Formally, the function $\psi$ (regret function [10]) represents the comparison between the value $(v)$ of choice (a) Partial feedback

(i)

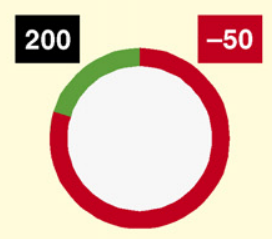

(ii)

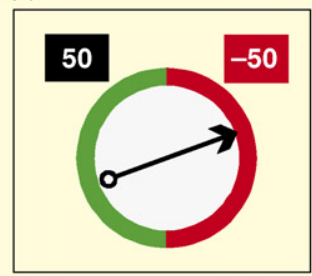

(b) Complete feedback

(i)

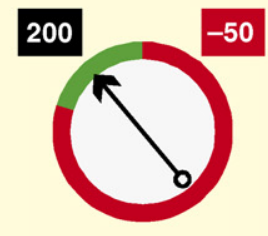

(ii)

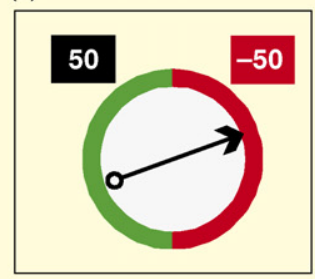

Disappointment

Figure 1. The regret gambling task. In the gambling task used in the studies by Camille et al. [5], Coricelli et al. [6] and Mellers et al. [7], subjects choose between two gambles (depicted as two 'wheels of fortune'). For instance, if subjects choose the gamble on the left [(i) in (a) and (b)], they might win 200 euros with $20 \%$ probability or lose 50 euros with $80 \%$ probability; if they choose the gamble on the right [(ii) in (a) and (b)], they might win or lose 50 euros with equal probabilities. There are two main contextual conditions in terms of the feedback provided - partial feedback and complete feedback. In partial feedback (a), only the outcome of the chosen gamble is provided, whereas in the complete feedback condition (b), both the outcome of the chosen and the unchosen gambles are provided. Complete feedback enables the subjects to judge not only the financial consequence of their choice, but also the outcome if they had they selected the other option (regret or relief). Emotional evaluations, physiological responses (skin conductance responses), choice behavior and brain activity are influenced by these different levels of feedback. Modified, with permission, from Ref. [5]. 
$(x)$ and the value of a rejected alternative $(y)$ :

$\psi[v(x)-v(y)]$

In regret theory, the function $\psi$ enters into the utility function $(U)$ :

$U(x, y)=v(x)+\psi[v(x)-v(y)]$

The utility of an act (e.g. choice of $x$ and simultaneous rejection of $y$ ) can be computed as the sum of the expected utility of the chosen act $(x)$ and of the anticipated regret term $\psi($.$) . These two terms are additive (i.e. value of an$ act $=$ expected utility + regret term $). \psi($.$) is decreasingly$ concave in the case of regret aversion $\left(\psi^{\prime \prime}<0\right.$, negative second derivative), which accords with the commonly observed pattern of choice behavior [7] (H. Bleichrodt et al., unpublished).

\section{The neural basis of regret}

One important question is whether the emotion of regret is subserved by specific cerebral regions. Camille et al. [5] studied the relationship between decision making and emotion in normal subjects and in patients with selective lesions to the OFC. The experimental task required subjects to choose between two gambles, each having different probabilities and different expected outcomes (Figure 1). Regret was induced by providing information regarding the outcome of the unchosen gamble. When subjects were asked to rate their emotional state after seeing the obtained outcome, normal controls reported emotional responses consistent with counterfactual thinking between obtained and non-obtained outcomes. Thus, a win of $\$ 50$ when the alternative gamble won $\$ 200$ induced a strong negative emotion. Conversely, the same outcome when confronted with a losing alternative gamble $(-\$ 200)$ created a feeling of relief. After being exposed to several trials in which they experienced regret, control subjects subsequently began to choose the gambles with probable outcomes likely to produce minimal regret, indicating that they had learnt from their prior emotional experience. Therefore, control subjects chose between risky gambles by a process that involved anticipating regret and maximizing expected values, thus integrating consideration about future emotional responses to the outcome of their choices.

By contrast, patients with lesions of the OFC did not report regret and did not anticipate negative affective consequences of their choices. They reported being happy when winning and disappointed when losing. Their emotional states were even modulated by the amount of the win $(+\$ 50$ or $+\$ 200)$ or the amount of the loss $(-\$ 50$ or $-\$ 200)$ but not by the value of the outcome in the alternative unchosen gamble. More striking, they persisted in choosing the gamble with a high probability of producing regret, and which normal subjects tended to avoid. Thus, patients with lesions to the OFC are unable to generate outcome evaluation and outcome expectancies, based upon a counterfactual comparison between the value of a chosen and a rejected alternative. Formally, they are unable to generate the regret function $\psi[10]$. Furthermore, the OFClesioned patients are not able to incorporate experienced regret into the process of choice behavior, and do not anticipate regret or learn from their regret-inducing decisions. Formally, these patients are unable to incorporate the function $\psi($.) into the decision-making process - that is, into their utility function. Patients with selective lesions in the OFC show a $\psi=0$; thus, they make their judgments solely on the basis of what has actually occurred, and do not experience and anticipate regret. Paradoxically, the behavior of OFC-lesioned patients is more in line with the prescriptions of rational theory, such that their behavior accords with maximization of expected utility. The absence of regret in OFC-lesioned patients suggests that these patients fail to grasp this concept of liability for one's own decisions that colors the emotion experienced by normal subjects. It is important to highlight the fact that OFC-lesioned patients are not emotionally unresponsive. For instance, these patients expressed a normal level of disappointment in the study by Camille et al. [5], and a higher than normal level of anger in response to unfairness in social situations (unfair offers in an Ultimatum Game) [13]. Thus, a key behavioral observation is that patients with lesions in the OFC are unable to experience regret and to anticipate the potential affective consequence of their choices.

We next review research that supports an assertion that the OFC: (i) exerts a top-down modulation of emotional processing as a result of a declarative process; (ii) integrates cognitive and emotional components of the entire (expectancies and evaluation) decision-making process; and (iii) thereby exerts a fundamental role in adaptive behavior.

\section{The OFC exerts a top-down modulation of emotions as a result of a declarative process}

Coricelli et al. [6] measured brain activity using functional magnetic resonance imaging (fMRI) while subjects participated in the regret gambling task (Figure 1).

Increasing regret was correlated with enhanced activity in the medial OFC region, the dorsal anterior cingulate cortex (ACC) and the anterior hippocampus (Figure 2). This hippocampal activity is consistent with the idea that a cognitive-based declarative process of regret is engaged by the task. We conjecture that hippocampal activity supports a modulation of declarative (consciously accessible) memory $[14,15]$, such that after a bad outcome, the lesson to be learnt is: 'in the future, pay more attention to the potential consequences of your choices'. Furthermore, Coricelli et al. [6] showed that activity in response to experiencing regret (processed in the OFC, ACC and medial temporal cortex) is distinct from activity seen with mere outcome evaluation (processed in the ventral striatum), and in disappointment elicited by the mismatch between actual and expected outcome of choice (processed in the middle temporal gyrus and dorsal brainstem). This suggests distinctive neural substrates in reward processing (Box 2), and the fact that the OFC and medial temporal cortex areas can bias basic dopamine-mediated reward responses [16].

OFC activity related to the level of responsibility in the process of choice (agency), and with the available information regarding alternative outcomes (regret), influences basic responses related to reward (monetary wins) and 


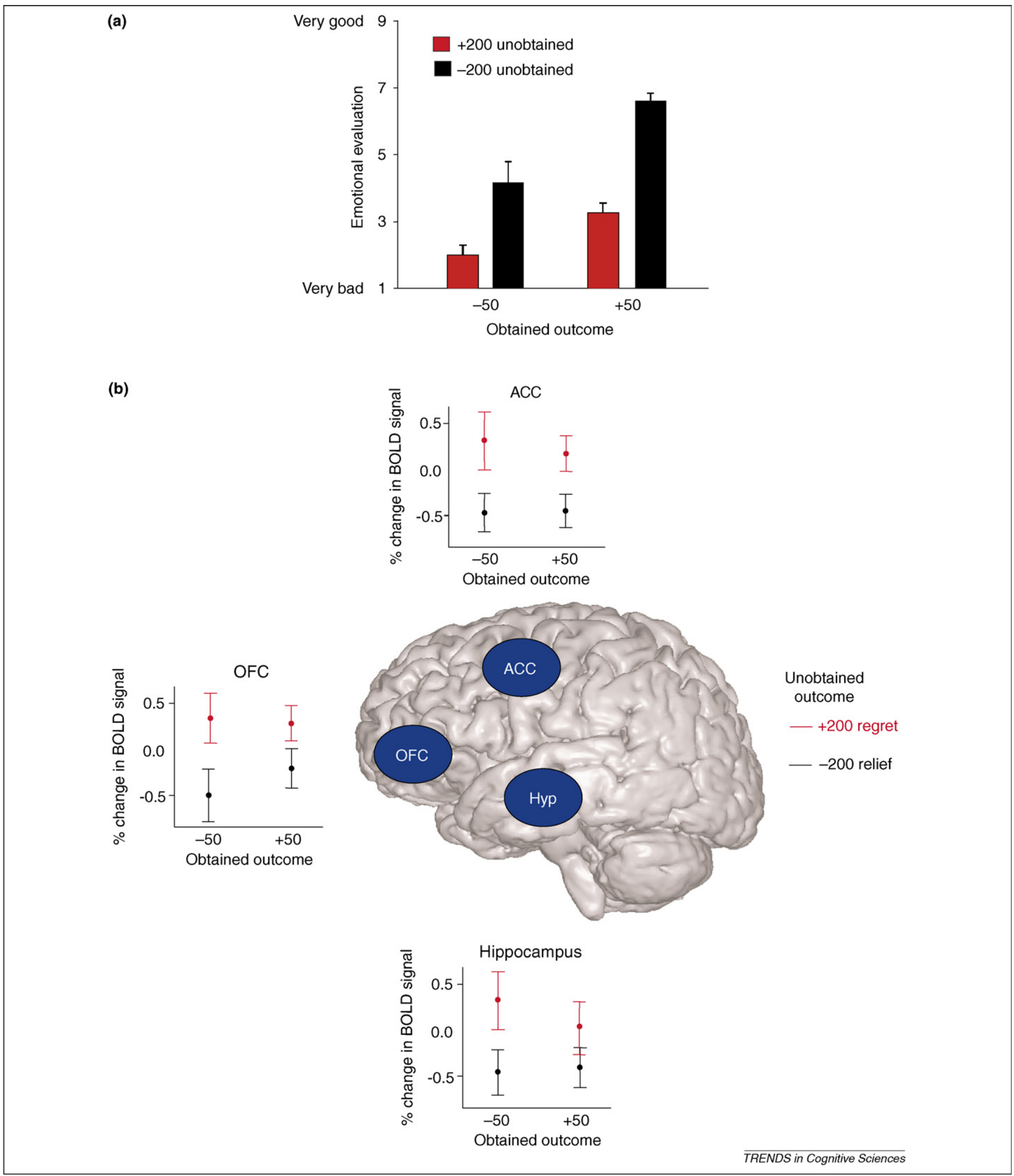

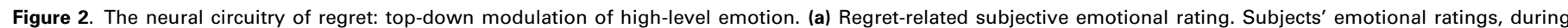
outcome evaluation, were a function of the foregone outcome $(+200$, regret; or -200 , relief). Losing 50 when the unchosen alternative wins 200 induces a strong negative feeling, whereas the same outcome $(-50)$ is perceived as less negative when the other gamble loses more $(-200)$. Interestingly, losing 50 when the alternative loses more $(-200)$ is considered as less negative than winning 50 when the outcome of the unchosen gamble is 200 . These results show how the unobtained outcome strongly

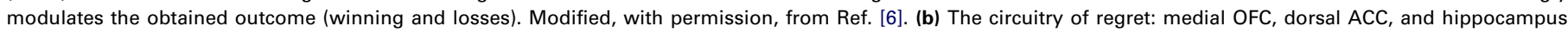
(Hyp). Regret-related activity in the ACC corresponds to the dorsal part of the ACC, thus in its cognitive division [48-50]. Hippocampal activity is consistent with the declarative component of regret. The interplay of those two areas (ACC and Hyp), in addition to the OFC, suggests that regret is elicited through a cognitive (top-down modulation) and declarative process. Modified, with permission, from Ref. [6]. 
Box 2. How the brain attributes affective values to alternatives of our choice

We distinguish two levels of reward processing in the brain.

\section{First-level reward processing}

First-level processing is based on signaling within dopaminergic neurons [53]. Dopamine (DA) is a neurochemical (that functions as a neurotransmitter) formed in midbrain neurons, such as the ventral tegmentum and the substantia nigra, which both send diffuse projections to areas including the striatum (basal ganglia) and OFC. The activity of these neurons is characterized by a (bottom-up) process of alert that expresses a teaching signal, representing an efficient basic learning mechanism (prediction error) [54-56]. Midbrain neurons differentiate rewarding from non-rewarding stimuli (punishers) in the environment [57], define expectations (perhaps through tonic activity), and detect a mismatch between expected and actual outcomes. Once a stimulus-response association is established, DA neurons are active only when the response is larger than expected, and deactivated when a response is unexpectedly unfavorable [53]. For first-level reward processing, the brain is not able to discriminate between different rewards (alternatives), which is the main point of economic decision making.

\section{Second-level reward processing}

Reward processing, belonging to the second level, is related to neuronal activity in regions such as the OFC, ACC, and, perhaps, the amygdala [58]. These responses are related to how we learn to select appropriate rewards on the basis of relative preferences $[18,27,59]$ or affective value $[60]$

Striato-OFC interaction: from the first- to second-level reward processing

First- and second-level reward processing is mutually interdependent. Indeed, reward information from the first level defines and updates relative reward values and subjective preferences. Put simply, the information within the dopamine reward responses (first level) enter into cognitive and emotional representations of reward in terms of subjective preferences and affective values. The neural systems (midbrain and basal ganglia) related to first-level reward processing have an adaptive function which is limited to facilitate (Go) responses that lead to positive outcomes (reward), or suppression (NoGo) of responses that lead to negative outcomes (punishers) [61]. The OFC can bias responses according to their relative reward and affective value (top-down modulation), approximating preferences and emotional experience of the organism. The medial temporal regions (e.g. $A B L$ ) support the OFC in assigning affective value to the choice outcomes, and update these associations within a representational memory. These two regions are strongly interconnected [62], and computational models show how the adaptive role of the amygdale-OFC system is much more flexible and is related to more complex behavior compared with dopamine-basal ganglia system $[19,61]$. This approach is consistent with the Goldman-Rakic [63] assertion that the crucial contribution of the prefrontal cortex in determining behavior involves situations where 'inner models of reality are used to govern behavior' (second level).

punishers (monetary losses). In several studies, medial OFC activity reflects reward attainment [17-19], whereas the lateral OFC is often associated with reversal learning, where subjects need to change behavioral strategies that are no longer advantageous [20-23]. This has been interpreted as suggesting that the medial OFC might support positive emotions, and the lateral OFC might support emotions with negative valence. Nevertheless, other neuroimaging studies [6] highlight a more complex role in reinforcement representations that is also suggested by lesion data [5] (lesions of the medial OFC do not impair the processing of primary rewards).

\section{The OFC integrates cognitive and emotional components during decision making}

An influential view (Damasio and co-workers [24-26]) restricts the role of the OFC to the anticipatory phase of decision making. However, we suggest that the OFC contributes to each and every phase of the process of choice. A seminal paper by Tremblay and Schultz [27] has demonstrated that neurons in the OFC of monkeys fire as a function of relative preference during instruction, expectation (sustained activation preceding reward) and delivery of reward. In humans, OFC activity is enhanced [18] when subjects build expectations over a risky gamble (prospect phase) and compute a comparison between different reward levels in the outcome phase when uncertainty is resolved. Coricelli et al. [6] found enhanced OFC activity when subjects experienced regret (outcome phase) and before making further choices (anticipating regret).

Thus, the OFC integrates cognitive and emotional components across the entire process of decision making; when it malfunctions, it results in behavior that is maladaptive in relation to ongoing contingencies.

\section{The OFC has a fundamental role in adaptive behavior}

Coricelli et al. [6] reported that, across their fMRI experiments, subjects became increasingly regret aversive, a cumulative effect reflected in enhanced activity within the ventromedial OFC and amygdala. Under these circumstances, the same pattern of activity that was expressed with the experience of regret was also expressed just before choice (Figure 3), suggesting that the same neural circuitry mediates both direct experience of regret and its anticipation. OFC activity related to the effect of experienced emotions connected with potential behavioral adjustment has also been found in a recent study by Beer et al. [28]. Thus, the OFC and amygdala contribute to this form of high-level learning based on past emotional experience, in a manner that mirrors the role of these structures in the acquisition of value in low-level learning contexts [19]. Indeed, animal [29] and human [30-33] neuroimaging studies assign a fundamental role to the amygdala in classical conditioning experiments, indicating its role in associative learning (acquiring cue outcome association). Schoenbaum et al. [34], recording in the OFC in rats with lesions in the basolateral amygdala (ABL), found loss of acquisition of associative information, although the process of outcome anticipation remained intact. This suggests that the ABL supplies value-related associative information to the OFC [35]. Conversely, lesions in the OFC of rats reduced ABL associative encoding ability, showing that OFC itself facilitates learning [36].

Moreover, the affective consequences of choice, such as the experience of regret, can induce specific mechanisms of cognitive control [37]. Coricelli et al. [6] observed enhanced responses in the right dorsolateral prefrontal cortex, right lateral OFC and inferior parietal lobule during a choice phase after the experience of regret [6] (Figure 3), and subsequent choice processes induced reinforcement, or avoidance, of the experienced behavior [38]. Corroborating results from Simon-Thomas et al. [39] show that negative emotions can recruit cognitive-based right hemisphere responses. Negative affective consequences 


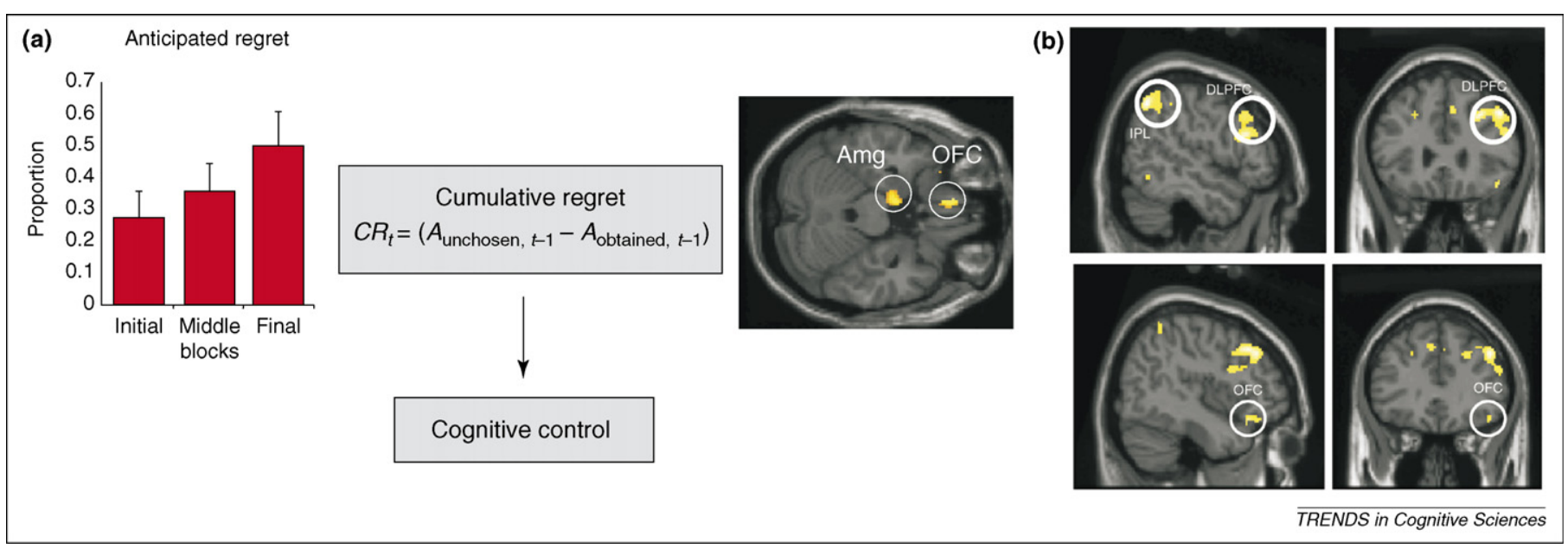

Figure 3. The neural basis of the adaptive function of regret. (a) The observed reactivation pattern of the OFC and amygdala (Amg) before subjects made their choices accounts for the behavioral impact. Across iterations of the experiment, subjects' behavior was more and more in line with a pattern that was explained in terms of regret avoidance, reflecting the 'cumulative' (learning) effect of experienced negative outcomes. The process of defining expectancies over possible consequences of the alternative of choice is based on an emotional reinforcement learning account. Cumulative regret (CR) is the difference between the average payoff realized and the average payoff missed (the payoff of the unselected gamble) over time. In other words, the impact of the consequences of rejected choices (regret) is indeed increasingly integrated in the process of choice through experience. Modified, with permission, from Ref. [6]. (b) Cognitive control activity induced by the experience of regret was observed during choices when the subjects just experienced regret $(t-1)$. Enhanced activity in the dorsolateral prefrontal cortex (DLPFC), parietal cortex (inferior parietal lobule, IPL) and right OFC is observed. Activity in the lateral OFC was also found at the time of outcome evaluation during regret and disappointment, suggesting that it might be responsible for the common process underlying the two emotions, and, thus, for counterfactual thinking. Coricelli et al. [6] found a pattern of reactivation based on the counterfactual thinking expressed at the moment of choice (ex ante comparison between the possible consequence of choice as part of the anticipated regret process) and during outcome evaluation. Such counterfactual reasoning enabled them to anticipate future regret, looking into the future consequences of each gamble. Modified, with permission, from Ref. [6].

(regret) induce specific mechanisms of cognitive control on subsequent choices.

\section{Theoretical foundation of regret-based adaptive processes}

Anticipation of regret induces a disposition to change behavioral strategies [40], and characterizes an emotional motivated learning process in decision making [41]. Recently, economists have proposed a class of learning rules which rely on regret. For instance, the 'regret matching' [42] is a simple behavioral procedure based not on best reply (i.e. Nash equilibrium) but on better replies to any possible action of others. Any better action can be chosen with a probability proportional to a measure of regret. In a dynamic setting (repeated games), the player needs to decide (at each trial) between keeping the strategy just played, and switching to other strategies. Based on a regret-matching procedure, the player changes his current strategy for a foregone alternative that would have given a higher payoff. Calling $U$ the player total payoff resulting from strategy $j$, and $V(k)$ the foregone payoff if strategy $k$ had been played every time in the past, then $V(k)-U$ represents the regret (if $V(k)>U$ ) of having played strategy $j$ instead of $k$. The probability of switching behavior is proportional to the value of this measure of regret. This simple adaptive (in the sense that it leads to flexible behavior) heuristic is a boundedly rational strategy, leading in the long run to a rational and sophisticated solution of the game (correlated equilibrium). Regret matching ( per $s e$ ) requires a low level of rationality [42], and is based on simple computations of the realized payoff (as the result of the used strategy) and the foregone payoffs of alternative strategies. Using the regret-matching rule, players do not need any information about the opponents' payoffs or actions [42], and the theory shows that convergence of a regret-matching rule to the correlated equilibria can arise also in the extreme case of the unknown game, in which players do not know the game and the choices of other players. This computation is dramatically less complex and demanding compared with somewhat unrealistic full rational optimization dynamics such as a belief-learning process (Bayesian learning), which requires updates at any time of the (prior) beliefs of others using Bayes' rule, and the definition of best replies, which chooses the optimal action given the beliefs [43].

\section{Synthesis and comments}

We suggest that the OFC integrates cognitive and emotional information - for example, under counterfactual processing that engenders a high-level emotion of regret. The OFC processes reward information and manipulates these data by ascribing affective and subjective values, the main determinants of human decision making. The OFC, through interactions with other brain areas, such as the amygdala, enables this region to provide an updated representation of value that can influence adaptive behavior. Recent work in economics provides a theoretical foundation for these neuroscientific findings. Thus, experimental and theoretical results demonstrate an adaptive role of the cognitive-based emotion of regret, and regret also figures prominently in the literature of learning in games. A remarkable result in this literature is that if players in a game minimize regret, the frequency of their choices converges to a correlated equilibrium (i.e. the rational solution) of the game [42] (D.P. Foster et al., unpublished). This has a general implication for our understanding of the role of emotions in decision making and rejects the dual or conflict view of 'emotion versus cognition' (rationality) [44] by showing the powerful consequences of full integration between these two components of human decision making. 


\section{Box 3. Outstanding questions}

- How can the dominant view in computational neuroscience of Bayesian (hyper-rational) functioning at a neuronal level and actual (less-than-rational) human behavior be reconciled?

- Do anticipatory versus anticipated processing dissociate at the neuronal level during decision making?

- Can maladaptive behaviors observed in psychiatric patients (e.g. schizophrenia or depression) be attributed to their inability to experience regret (schizophrenia) or an overexpression of regret (depression)?

- Do cognitive-based emotions, such as regret and guilt, have a common neural circuitry?

\section{Perspectives}

Economists have produced remarkable data describing how we make economic choices. The 'rational choice' approach has been enormously successful at yielding a simplified theory of the brain processes underlying economical strategies. Recently, the introduction of neuroscience tools (brain imaging techniques, lesion studies, single-cell recording in non-human primates) and increasing evidence as to the importance of emotional and social states in economic decision making are opening new perspectives in the field of neuroeconomics [45-47]. We expect this initiative to foster a new interface between theory and experimentation, between mathematical models and brain function. Some of the questions that are still outstanding are listed in Box 3. Neurobiological approaches can contribute greatly to a better understanding of the cognitive and emotional underpinnings of economic decision making, including how people evaluate and anticipate rewards, through to how they form beliefs about what other people feel or might do. Reciprocally, the mathematical formalism of economic theories provides new ways of analyzing the neural computations involved in representing decision variables such as anticipated regret.

\section{Acknowledgements}

We gratefully acknowledge financial support from the Human Frontier Science Program (HFSP, RGP 56/2005) to G.C. and A.S.; and the Wellcome Trust Programme Grant to R.J.D.

\section{References}

1 Roese, N.J. and Olson, J.M. (1995) What Might Have Been: The Social Psychology of Counterfactual Thinking. Erlbaum

2 Byrne, R.M. (2002) Mental models and counterfactual thoughts about what might have been. Trends Cogn. Sci. 6, 426-431

3 Zeelenberg, M. and van Dijk, E. (2004) On the comparative nature of the emotion regret. In The Psychology of Counterfactual Thinking (Mandel, D. et al., eds), Routledge

4 Von Neumann, J. and Morgenstern, O. (1944) Theory of Games and Economic Behavior. Princeton University Press

5 Camille, N. et al. (2004) The involvement of the orbitofrontal cortex in the experience of regret. Science 304, 1167-1170

6 Coricelli, G. et al. (2005) Regret and its avoidance: a neuroimaging study of choice behavior. Nat. Neurosci. 8, 1255-1262

7 Mellers, B. et al. (1999) Emotion-based choice. J. Exp. Psychol. Gen. 3, $332-345$

8 Frijda, N.H. (1986) The Emotions, Cambridge University Press

9 Kahneman, D. and Miller, D. (1986) Norm theory: comparing reality to its alternatives. Psychol. Rev. 93, 136-153

10 Bell, D.E. (1982) Regret in decision-making under uncertainty. Oper. Res. 30, 961-981
11 Loomes, G. and Sugden, R. (1982) Regret theory: an alternative theory of rational choice under uncertainty. Econ. J. 92, 805-824

12 Gilovich, T. and Melvec, V.H. (1994) The temporal pattern to the experience of regret. J. Pers. Soc. Psychol. 67, 357-365

13 Koenigs, M. and Tranel, D. (2007) Irrational economic decision-making after ventromedial prefrontal damage: evidence from the Ultimatum Game. J. Neurosci. 27, 951-956

14 Eichenbaum, H. (2004) Hippocampus: cognitive processes and neural representations that underlie declarative memory. Neuron 44, 109 120

15 Steidl, S. et al. (2006) Effects of emotional arousal on multiple memory systems: evidence from declarative and procedural learning. Learn. Mem 13, 650-658

16 De Martino, B. et al. (2006) Frames, biases, and rational decisionmaking in the human brain. Science $313,684-687$

17 Rolls, E.T. (2000) The orbitofrontal cortex and reward. Cereb. Cortex $10,284-294$

18 Breiter, H.C. et al. (2001) Functional imaging of neural responses to expectancy and experience of monetary gains and losses. Neuron 30, 619-639

19 Gottfried, J.A. et al. (2003) Encoding predictive reward value in human amygdala and orbitofrontal cortex. Science 301, 11041107

20 Elliott, R. et al. (2000) Dissociable functions in the medial and lateral orbitofrontal cortex: evidence from human neuroimaging studies. Cereb. Cortex 10, 308-317

21 Fellows, L.K. and Farah, M.J. (2003) Ventromedial frontal cortex mediates affective shifting in humans: evidence from a reversal learning paradigm. Brain 126, 1830-1837

22 Hornak, J. et al. (2004) Reward-related reversal learning after surgical excisions in orbito-frontal or dorsolateral prefrontal cortex in humans. J. Cogn. Neurosci. 16, 463-478

23 O'Doherty, J. et al. (2001) Abstract reward and punishment representations in the human orbitofrontal cortex. Nat. Neurosci. 4, 95-102

24 Damasio, A.R. (1994) Descartes' Error: Emotion, Reason, and the Human Brain. Putnam

25 Bechara, A. et al. (2000) Emotion, decision making and the orbitofrontal cortex. Cereb. Cortex 10, 295-307

26 Bechara, A. et al. (2000) Characterization of the decision-making deficit of patients with ventromedial prefrontal cortex lesions. Brain 123, 2189-2202

27 Tremblay, L. and Schultz, W. (1999) Relative reward preference in primate orbitofrontal cortex. Nature 398, 704-708

28 Beer, J.S. et al. (2006) Controlling the integration of emotion and cognition: the role of frontal cortex in distinguishing helpful from hurtful emotional information. Psychol. Sci. 17, 448-453

29 Amorapanth, P. et al. (2000) Different lateral amygdala outputs mediate reactions and actions elicited by a fear-arousing stimulus. Nat. Neurosci. 3, 74-79

30 LaBar, K.S. et al. (1998) Human amygdala activation during conditioned fear acquisition and extinction: a mixed-trial fMRI study. Neuron 20, 937-945

31 Buchel, C. et al. (1998) Brain systems mediating aversive conditioning: an event-related fMRI study. Neuron 20, 947-957

32 LaBar, K.S. and Cabeza, R. (2006) Cognitive neuroscience of emotional memory. Nat. Rev. Neurosci. 7, 54-64

33 O'Doherty, J.P. et al. (2002) Neural responses during anticipation of a primary taste reward. Neuron $33,815-826$

34 Schoenbaum, G. et al. (2003) Encoding predicted outcome and acquired value in orbitofrontal cortex during cue sampling depends upon input from basolateral amygdala. Neuron $39,855-867$

35 Schoenbaum, G. et al. (2003) Lesions of orbitofrontal cortex and basolateral amygdala complex disrupt acquisition of odor-guided discriminations and reversals. Learn. Mem. 10, 129-140

36 Saddoris, M.P. et al. (2005) Rapid associative encoding in basolateral amygdala depends on connections with orbitofrontal cortex. Neuron 46, 321-331

37 Yarkoni, T. et al. (2005) Sustained neural activity associated with cognitive control during temporally extended decision making. Brain Res. 23, 71-84

38 Clark, L. et al. (2004) The neuropsychology of ventral prefrontal cortex: decision-making and reversal learning. Brain Cogn. 55, 41-53 
39 Simon-Thomas, E.R. et al. (2005) Behavioral and electrophysiological evidence of a right hemisphere bias for the influence of negative emotion on higher cognition. J. Cogn. Neurosci. 17, 518-529

40 Ritov, I. (1996) Probabilities of regret: anticipation of uncertainty resolution in choice. Organ. Behav. Hum. Decis. Process. 66, 228-236

41 Zeelenberg, M. et al. (1996) Consequences of regret aversion: effects of expected feedback on risky decision making. Organ. Behav. Hum. Decis. Process. 65, 148-158

42 Hart, S. and Mas-Colell, A. (2000) A simple adaptive procedure leading to correlated equilibrium. Econometrica $68,1127-1150$

43 Kalai, E. and Lehrer, E. (1993) Rational learning leads to Nash equilibrium. Econometrica 61, 1019-1045

44 Sanfey, A.G. et al. (2006) Neuroeconomics: cross-currents in research on decision-making. Trends Cogn. Sci. 10, 108-116

45 Camerer, C.F. (2003) Psychology and economics. Strategizing in the brain. Science 300, 1673-1675

46 Glimcher, P.W. and Rustichini, A. (2004) Neuroeconomics: the consilience of brain and decision. Science 306, 447-452

47 Rustichini, A. (2005) Neuroscience. Emotion and reason in making decisions. Science 310, 1624-1625

48 Bush, G. et al. (2000) Cognitive and emotional influences in anterior cingulate cortex. Trends Cogn. Sci. 4, 215-222

49 Carter, C.S. et al. (1999) The contribution of the anterior cingulate cortex to executive processes in cognition. Rev. Neurosci. 10, 49-57

50 Critchley, H.D. (2004) The human cortex responds to an interoceptive challenge. Proc. Natl. Acad. Sci. U. S. A. 101, 6333-6334

51 Allais, M. (1953) Le comportement de l'homme rationnel devant le risque: critique des postulats et axiomes de l'école américaine. Econometrica 21, 503-546

52 Kahneman, D. and Tversky, A. (1979) Prospect theory: an analysis of decision under risk. Econometrica 47, 263-292
53 Schultz, W. (2002) Getting formal with dopamine and reward. Neuron $36,241-263$

54 Schultz, W. et al. (1997) A neural substrate of prediction and reward. Science 275, 1593-1599

55 Hollerman, J.R. and Schultz, W. (1998) Dopamine neurons report an error in the temporal prediction of reward during learning. Nat. Neurosci. 1, 304-309

56 Schultz, W. (1998) Predictive reward signal of dopamine neurons. J. Neurophysiol. 80, 1-27

57 Tobler, P.N. et al. (2005) Adaptive coding of reward value by dopamine neurons. Science 307, 1642-1645

58 O'Doherty, J.P. (2004) Reward representations and reward-related learning in the human brain: insights from neuroimaging. Curr. Opin. Neurobiol. 14, 769-776

59 Padoa-Schioppa, C. and Assad, J.A. (2006) Neurons in the orbitofrontal cortex encode economic value. Nature 441, 223-226

60 Kringelbach, M.L. (2005) The human orbitofrontal cortex: linking reward to hedonic experience. Nat. Rev. Neurosci. 6, 691-702

61 Frank, M.J. and Claus, E.D. (2006) Anatomy of a decision: striatoorbitofrontal interactions in reinforcement learning, decision making and reward. Psychol. Rev. 113, 300-326

62 Cavada, C. et al. (2000) The anatomical connections of the macaque monkey orbitofrontal cortex. A review. Cereb. Cortex 10, 220-242

63 Goldman-Rakic, P.S. (1995) Architecture of the prefrontal cortex and the central executive. Ann. N. Y. Acad. Sci. 769, 71-83

64 Aumann, R.J. (1974) Subjectivity and correlation in randomized strategies. J. Math. Econ. 1, 67-96

65 Nash, J.F. (1950) Equilibrium points in n-person games. Proc. Natl. Acad. Sci. U. S. A. 36, 48-49

66 Kreps, D.M. (1990) A Course in Microeconomic Theory. Princeton University Press

\section{Elsevier.com - linking scientists to new research and thinking}

Designed for scientists' information needs, Elsevier.com is powered by the latest technology with customer-focused navigation and an intuitive architecture for an improved user experience and greater productivity.

The easy-to-use navigational tools and structure connect scientists with vital information - all from one entry point. Users can perform rapid and precise searches with our advanced search functionality, using the FAST technology of Scirus.com, the free science search engine. Users can define their searches by any number of criteria to pinpoint information and resources. Search by a specific author or editor, book publication date, subject area - life sciences, health sciences, physical sciences and social sciences - or by product type. Elsevier's portfolio includes more than 1800 Elsevier journals, 2200 new books every year and a range of innovative electronic products. In addition, tailored content for authors, editors and librarians provides timely news and updates on new products and services.

Elsevier is proud to be a partner with the scientific and medical community. Find out more about our mission and values at Elsevier.com. Discover how we support the scientific, technical and medical communities worldwide through partnerships with libraries and other publishers, and grant awards from The Elsevier Foundation.

As a world-leading publisher of scientific, technical and health information, Elsevier is dedicated to linking researchers and professionals to the best thinking in their fields. We offer the widest and deepest coverage in a range of media types to enhance cross-pollination of information, breakthroughs in research and discovery, and the sharing and preservation of knowledge.

\section{Elsevier. Building insights. Breaking boundaries. www.elsevier.com}

